\title{
Interpretation of Transferred Epithet by Means of Conceptual Integration Theory
}

\author{
Xinmei Wang \\ School of Foreign Languages, Shandong University of Finance and Economics, Shandong, 250014, China

\begin{abstract}
Transferred epithet is one of the figures of speech frequently used in our expression. A better understanding of this seemingly illogical modification is undoubtedly of great benefit for common readers. In this paper, the conceptual integration theory advanced by Fauconnier and Turner $(1994,1997)$ is applied to the interpretation of transferred epithet. The purpose is to test the effectiveness of the blending framework, and this study is also expected to come up new insights to widen the wealth of data that is necessary to verify the generality of conceptual integration.
\end{abstract}

Index Terms - transferred epithet, conceptual integration, blending framework

\section{INTRODUCTION}

Figures of speech, as a kind of language form, possesses specific rhetoric effect and is creatively used in specific context, and it is also considered to be "a departure from the 'linguistic norms' of everyday language in some way, whether semantically or syntactically" (Wales, 1989: 170-177). While it has always been of interest of the rhetorician and is also considered peripheral phenomenon and outside of the core data which the linguists should be concerned (Coulson, 1997). Actually it is a pervasive phenomenon in every language as a kind.

Transferred epithet is one of the figures of speech frequently used in our expression. In rhetoric, the transferring in descriptive modifiers is a "betrayal" of the regular grammar. In literary works, it is used to create a kind of beauty with haziness, terseness and a dramatic sense. A better understanding of this illogical modification is undoubtedly of great benefit for common readers. Most of this rhetorical device focused on its aesthetic function. Unfortunately they failed to scientifically illustrate the concrete cognitive process of its meaning construction.

This paper gives an introduction to a new cognitive theory about building the semantic structure and semantic meaning, that is, conceptual integration theory advanced by Fauconnier and Turner (1996), and its conceptions of mental space, blending, on-line process and emergent structure, etc. With this theory, on one hand, the problems remained unsolved, that is, the meaning construction of transferred epithet can be fully explained, on the other hand, the generality of this theory is developed and its adequacy and descriptive power are tested.

\section{TRANSFERRED EPITHET}

Figures of speech are very effective and expressive in achieving balance between the obvious and the obscure, so that the readers could notice the ideas immediately and therefore accept them. Figures of speech can make our thoughts vivid and concrete. They help us to exchange ideas with others in a clear and effective way, because they stir emotional responses, they can carry truth and keep alive into the heart by passion. The transferred epithet is one of the rhetorical devices frequently used in our expressions. In most cases, the semantic transference in natural language relies on the use of transferred epithet, and transferred epithet is a universal semantic phenomenon (Leech, 1981).

\section{A. Definition of Transferred Epithet}

As an effective and specific rhetorical device, a series of definitions of transferred epithet are given by scholars.

A Dictionary of Literary Terms (Cuddon, J. A, 1979:315) thought that "transferred epithet is a figure of speech in which the epithet is transferred from the appropriate noun to modify another to which it does not really belong".

In Manual of English Grammar and Composition, Nesfield (1964: 284) defined it like this: When two impressions are associated together in the mind, an epithet that property belongs to one only is transferred to the other. One of the commonest, though not only, application of this figure is when some personal quality is transferred to something inanimate.

Lanham (1991:86) in a manual of Rhetoric Terms said "transferred epithet is a change in the relation of words by which a word, instead of agreeing with the case it logically qualifies, is made grammatically with another case".

Although we have various definitions about transferred epithet, they share more similarities than differences, that is, they share the same character, a transferred epithet, as its name implies, a figure of speech where a modifier (an adjective, the present and past participles, prepositional phrase, nouns or descriptive phrase) is transferred from the modified it should rightly modify to another which it should not modify or belong under the condition that the two 
impressions are closely associated. The following are some typical examples are: the scathing column, the baking court, a laughing and cheering ring, etc..

\section{B. The Process of Forming the Transferred Epithet}

The process for the forming of semantic interaction of transferred epithet consists of, first, the conflicting words between the modifier and the modified joining in the linear way; second, the exchanging and rearranging of the semantic meaning in the pre-positioned language situation, the interaction of which is the typical distinguishing characteristics for transferred epithet. Usually the form of transferred epithet is the modifier (adj., participles, descriptive phrases, "of" phrases, etc.) plus the modified (usually n.), and the literal meaning of the modifier and the modified obviously conflict to each other. For instance: silent moon. In the above example, the moon has the property of human beings, but they joined together.

\section{The Characteristics of Transferred Epithet}

The characteristics of transferred epithet can be considered in terms of its usage and effect of expression. On one hand, in transferred epithet, the modifier and the modified exist in context, out of context or in the counterfactual cognitive context. The semantic interaction of them is the typical distinguishing characteristic for transferred epithet. For example, Englishman likes to have a leisurely drink in a local pub. Leisurely and drink both has its own semantic set. According to their logical relation and association between them, we form the image relation and then semantic relation. The media for leisurely and drink is person. Their original logic is hidden and the illogical arrangement is foregrounded. Therefore, we have leisurely drink. On the other hand, from the point of view of its effect, transferred epithet makes language concise and comprehensive, vivid and vigorous, and also gives novel and original meaning. It helps reader to grasp the author's original idea and real intention.

Transferred epithet is more dynamic and vivid in communication and is full of inspiration. It is considered to be the typical picturesque and informative expression. It is worth noticing that, sometimes the modifier or the modified is hidden in the fictitious language space. That is, they do not appear in the sentence side by side. In this case, we are hoped to reestablish the hidden modifier or the context of modified to understand the transferred epithet smoothly. So the cognitive ability and the level of language have great influence in the understanding of rhetorical language.

\section{Mental Space AND CONCEPTUAL INTEGRATION THEORY}

\section{A. Mental Space}

The theory of mental spaces was developed in reaction to mainstream views of meaning. In recent years, many aspects of language that were not initially linked to mental space construction have been studies by scholars of different disciplines, and integrated into a more general powerful theory, conceptual integration theory. The mental space theory suggests that construction of meaning is neither static and nor permanent. Different projections, category assignments, and space configurations are stimulated locally in given situations.

On Fauconnier's (1994) model, "a mental space contains a partial representation of the entities and relations of a particular scenario as perceived, imagined, or remembered by a speaker. This representation typically includes elements to represent each of the discourse entities, and simple frames to represent the relationships that exist between them). Speakers set up spaces in order to partition the information evoked by a series of mental spaces and connections between them" (Fauconnier, 1994). That is, "mental spaces are small conceptual packets constructed as we think and talk, for purpose of local understanding and action. They are very partial assemblies containing elements, and structured by frames and cognitive models. They are interconnected and can be modified as thought and discourse unfold, and it can be used generally to model dynamic mappings in thought and language" (Fauconnier, 1994, 1997; Fauconnier \& Turner, 1996).

\section{B. Conceptual Integration}

Conceptual integration is a general cognitive operation used to construct meaning developed by Fauconnier (1997), and Fauconnier \& Turner (1998). It interacts with other general cognitive operation. It yields products that frequently become entrenched in conceptual structure and grammar (Fauconnier, 1997). It often performs new blending on its entrenched products. It is easy to detect in spectacular cases but it is for the most part a routine, workday process that escapes detection except on the technical analysis (Fauconnier, 1997). The essence of the operation is to construct a partial match between input mental spaces and to project selectively from those inputs into a novel 'blended' mental space, which then dynamically develops emergent structure (Fauconnier, 2003). It operates on two input mental spaces to yield a third space, the blend which inherits partial structure from the input spaces and has emergent structure of its own (Fauconnier, 2003). The following are some of the conditions which are satisfied when two input spaces input I1 and input $\mathrm{I} 2$ are blended:

(1) Cross-space Mapping: there is a partial mapping of counterparts between the input space 1 (input I1) and input space 2 (input I2).

(2) Generic Space: there is a generic space, which maps onto each of the inputs. This generic space reflects some common, usually more abstract structure and obligation shared by the inputs, and defines the core cross-space mapping 
between them. A generic space does not have to be available prior to the construction of a network. It is often constructed and projected along with other spaces and connections.

(3) Blend: the input I1 and input I2 are partially projected onto a fourth space, the blend.

(4) Emergent structure: the blend has emergent structure not provided by the inputs. This happens in three interrelated ways. We can see its full picture in fig. 1.

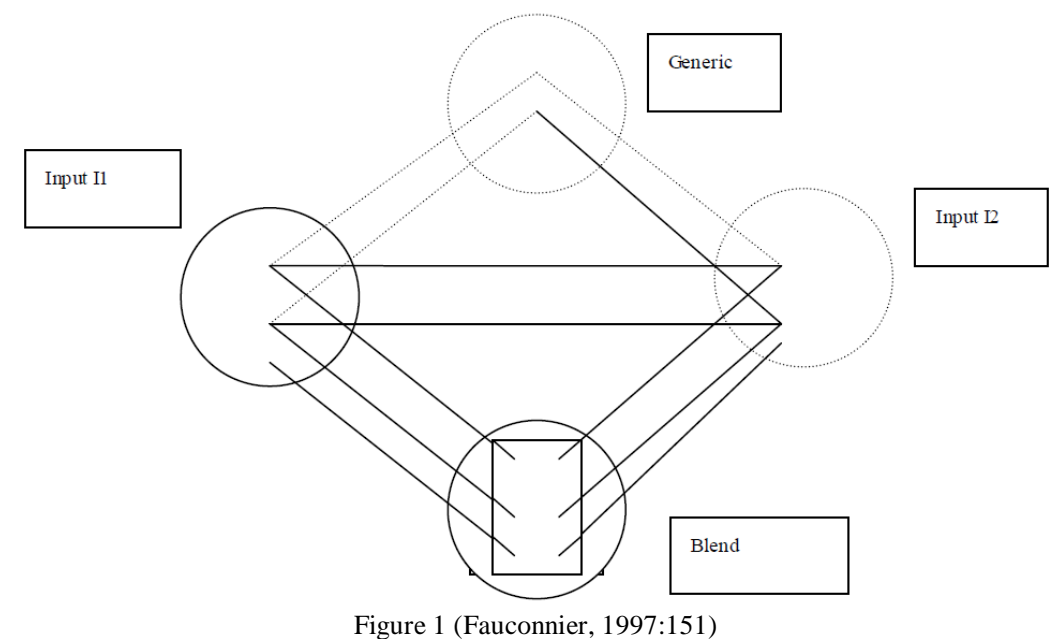

Conceptual integration involves the temporary construction of simple cognitive models and the establishment of cognitive mappings between different spaces (Fauconnier, 1997). The mapping, or mental space connection, can be understood as that an object or element in one space connects to an object or element in another space, and can be based on identity, similarity, metonymy or analogy.

\section{Blending Process}

Blending involves three processes, composition, completion and elaboration, each of which provides for the possibility of emergent structure (Fauconnier, 1997). Composition involves attributing a relation from one space to an element or elements from the input spaces. Emergent structure in composition arises from contextual accommodation of a concept from one domain to apply to elements in a different domain (Fauconnier, 1997). Completion is pattern completion which occurs when structure projected from the inputs matches information in long-term memory (Fauconnier, 1997).

We choose to compose structure from input spaces into the blend. That is, we apply counterpart connections between the input spaces. Thus, a working space for further composition became possible. Completion renders additional structure when certain elements have been taken into consideration. Elaboration develops the blend through imaginative mental simulation according to principles and logic in the blend (Fauconnier, 1997). Some of these principles will be brought to the blend by completion. Continued dynamic completion can recruit new principles and logic during elaboration. But new principles and logic may also arise through elaboration itself (Fauconnier, 1997).

\section{APPLICATION OF CONCEPTUAL INTEGRATION THEORY TO INTERPRETAION OF TRANSFERRED EPITHET}

Conceptual integration occurs at many levels of abstraction and in all sorts of contexts. In order to demonstrate the general utility of conceptual integration networks and in the light of the previous introduction, conceptual integration theory is applied to the integration of transferred epithet. The study is carried out from aspects of cross-mapping and integration networks.

\section{A. Transferred Epithet as Data of this Study}

There are several reasons which make me choose transferred epithet as data of this study. Firstly, traditional interpretation of it lays much emphasis on its aesthetic function rather than its cognitive operations involved in its meaning construction. Secondly, conventional study tends to interpret it in a rather static way, while language now is considered to be the human cognitive capacity, and actually we construct meaning dynamically. So this dissertation choose transferred epithet as data to give a new interpretation in dynamic way. Thirdly, the characteristics of transferred epithet make the application of conceptual integration theory feasible, because the cognitive interpretation of transferred epithet involves leaps between different mental spaces.

According to its definitions and characteristics, the following examples will be taken to give an illustration.

(1) The big man crashed down on a protesting chair.

(2) Her panic gave place to anger and a burning humiliation.

(3) The Grape of Wrath

(4) The boat blew a long mournful whistle into the mist. 


\section{B. Cross-space Mapping in Transferred Epithet}

Various researches in cognitive science prove that mapping across cognitivespaces plays a central role in language and thought. All blending depends upon cross-space mapping. Mappings between domains and blending are at the center of meaning construction and of the unique human cognitive capacity of producing, transferring and processing meaning.

\section{Analogical Mapping}

Phrase protesting chair in example (1) shows the characteristics of analogical mapping. Protesting is usually used to modify human who desires to express his disagreement, feeling of unfairness, annoyance, especially when he/she cannot withstand the heavy burden or dissatisfaction. The sentence also provides us a context in which the man with heavy weight falls on the chair violently and noisily. It seems impossible for chair to protest, but one property of 'protest' which exists in mind of both speaker and reader is its impassioned and vehement sound, and the chair will certainly cackle and creak under the heavy load. So they share a strong resemblance in the sound they make. Therefore, the phrase constructs a blend. 'The protesting man' comprises one input mental space (input I1). 'The chair under the heavy load' comprises the other input mental space (input I2). The generic space 'sound producing' defines the cross-space mappings between the two input spaces. They are the burden, the sound, the content, the way, expression of the protest, and the heavy load, the cackling or creaking of the chair. Which element in the input I1 is projected to the blend relies on the element projected to the blend in input I2. The other projections from input I1 space unrelated to the sound are omitted by the projected structure of heavy load, creaking and cackling of chair from the input I2, that is, the structure in input I1 that is projected to the blend is the burden, the impassioned and vehement sound of protesting. It is by analogical mapping that the two structures are projected to the blend. The analogical mapping is initiated by the structure of 'people protests' and 'chair creaks'. In blending, the emergent structure through composition is that the chair cannot withstand the heavy load. The emergent structure is completed by the context provided by the sentence is that the chair is protesting. Therefore, this combination protesting chair displays the vivid and humorous effect of figurative language. Figure 1 represents the conceptual integration network for the concept of protesting chair. See Fig.2.

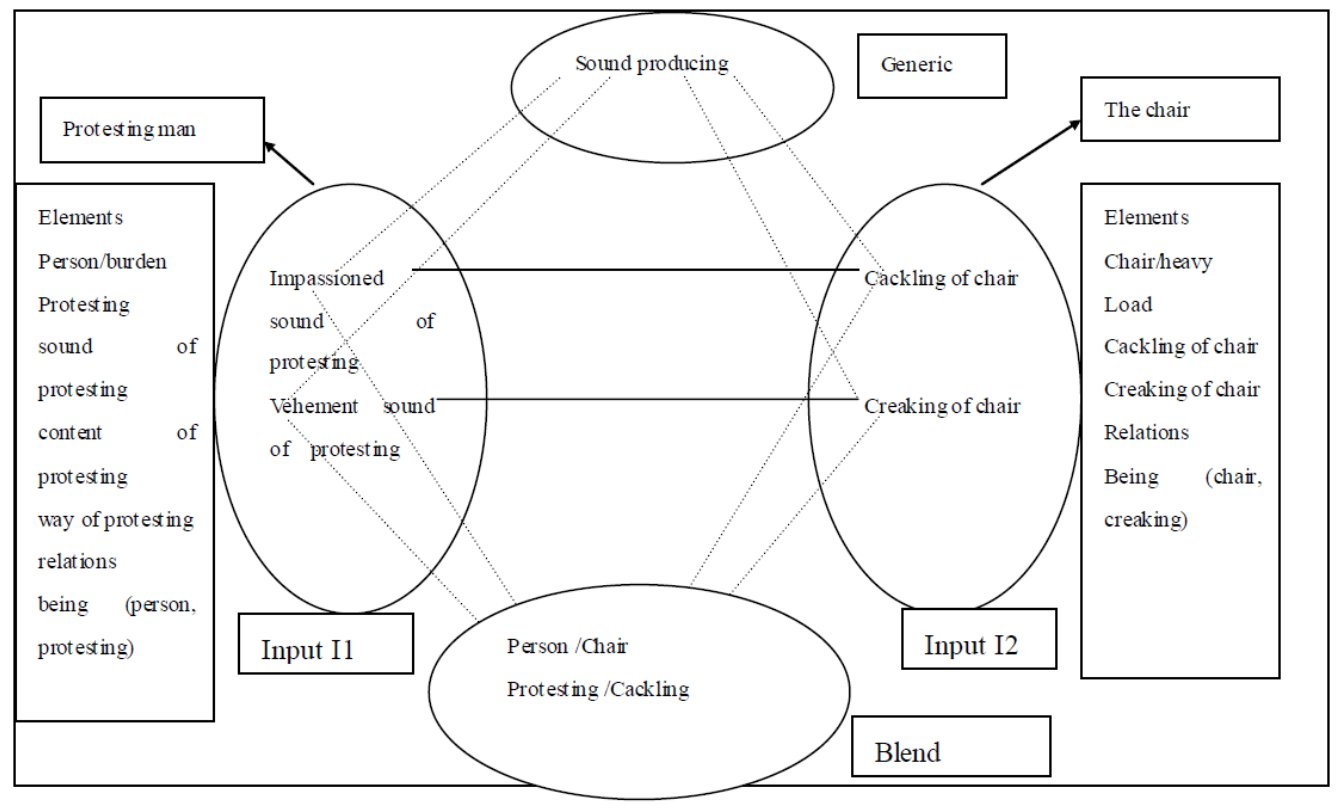

Figure 2

\section{Metaphorical Mapping}

In transferred epithet (2), the modifier burning conveys a concrete concept. Compared with burning, humiliation is a more abstract concept. From this phrase, we can elicit two input spaces. Burning which evokes one input space (input I1), presents the fire, while humiliation comprise the other input space (input I2). The cross-space mapping between the inputs is metaphorical, with fire as source input and humiliation as the target. This analysis reveals the required mapping between folk models of heat and folk model of humiliation. In this mapping, the fire maps onto an angry individual, smoke or steam (a sign of heat ) maps onto signs of humiliation, explosion (uncontrolled fire) maps onto extreme, uncontrolled humiliation. The important basis for this mapping is the physiological effects of humiliation: increased body heat, blood pressure, anger, redness in face, and so on. In the blended space, we find the individual and their emotions projected either from the source input of physical heat, explosion or from the body physiology linked to the emotion. Naturally, the generic space represents abstract commonalities between inputs: rising temperature. In blending, the emergent structure through composition is that fire is burning and he is also burning with rage. The emergent structure completed by the encyclopedic knowledge is he structure that humiliation tends to be out of control. 
3. Metonymical Mapping

Transferred epithet (3) is one kind of transferred epithet. It is also the name of novel written by John Steinbeck. With the Great Depression as its background, the book described that enormous farmers' land was forcibly occupied by the monopoly capitalists. What is waiting for the farmers was only the bankruptcy. In order to flee from famine, they had to move to the west. Unfortunately, they felt into another predicament.

Fig. 3 represents the conceptual integration network for the concept of grape of wrath. Here is how the integration network works. From this phrase, we can construct two input spaces. Wrath is the emotion of human beings. One of the input (input I1) mental spaces is the domain of emotion. Grape is a kind of fruit, so the domain of fruit comprises the second input mental space (input I2). Input I1 presents the structure of human emotions such as wrath, anger, happiness, excitement. While input $\mathrm{t}$ I2 presents the structure of planting grapes, taking care of grapes and picking grapes. There is a cross-space mapping between the two input spaces. The projection is partial and selective. In input I1, the emotion of wrath is projected to the blend. In input $\mathrm{I} 2$, the processes of growing grapes are projected to the blend. The generic space guiding this mapping contains an abstract event structure: agent and patient. In the blending, the emergent structure through composition is that people plant and pick fruits with some kind of emotion. While the emergent structure is completed by metonymical projection from wrath to grape. The emergent structure through completion is that the farmers plant and pick the grape wrathfully, that is, it is the farmers who got angry instead of the grape, which is also the theme of the novel.

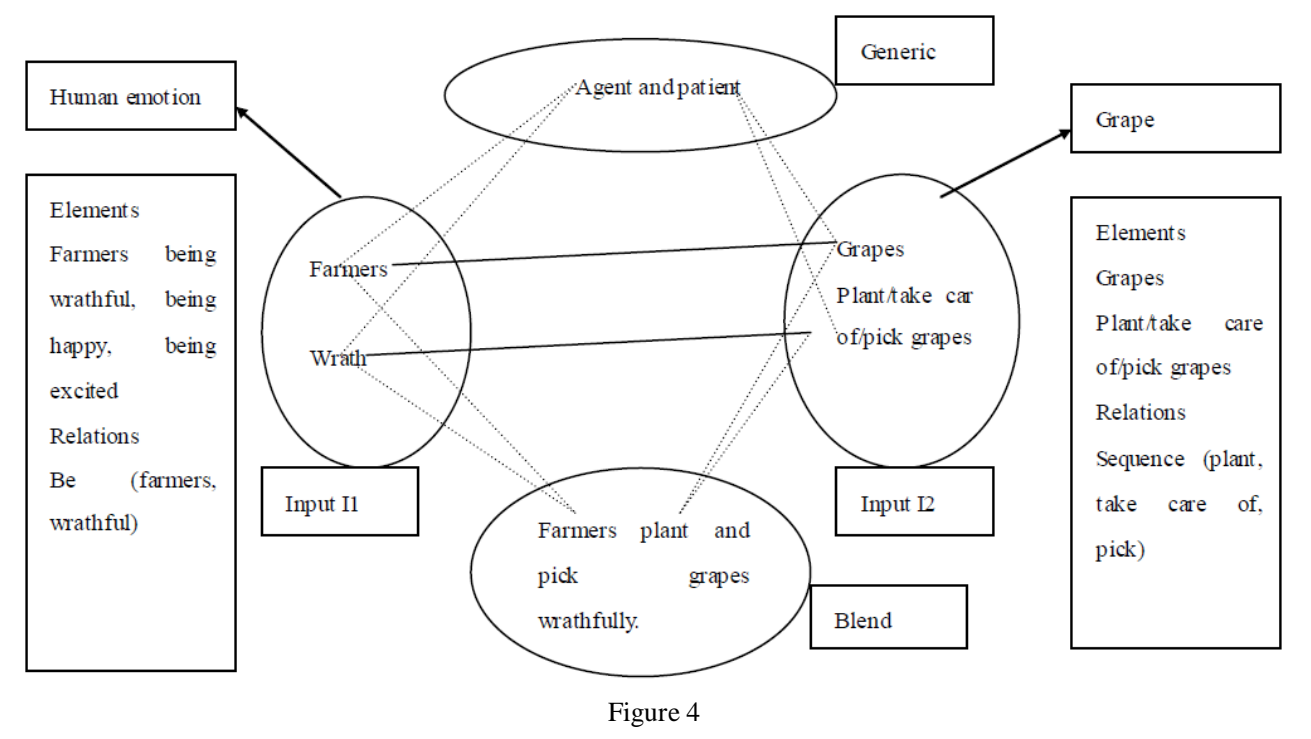

4. Mapping with the Phrase as One Input

In sentence (4), the phrase mournful whistle is a typical transferred epithet. Mournful which is used to modify person is now transferred to modify whistle. It is extracted from short story Eveline written by James Joyce. In this story the heroine Eveline was dispatched willfully at work, and had to face her fierce father at home. She had no alternative but living an empty and lifeless life. Later she got chance to escape from the strained and depressing circumstances and to pursue her own happiness with her boyfriend, but before they got on board ship, she lost all her courage and hesitation occupied her mind. The boat blew a long whistle into the mist. Her boyfriend as well as her happiness went far away. At that moment, she was fully filled with grief and sorrow. What she could do is just crying mournfully. The sentence conveyed two aspects of information: The boat blew a long whistle into the mist; the whistle sounds mournful when it blew. Instead of describing the heroine's mood directly, the author transferred mournful to the seemingly irrelevant word whistle. In this way, the reader experienced not only the mournful state of mind of the heroine but also the bitter atmosphere set off by the mist. The transferred epithet helps the sentence achieve the artistic effect of prominence through contrast. The following is how the integration network works.

In this network, there is an input space (input I1), structure with the state of mind of human being, the other input space (input I2) structure of boat, and a blended space with a cognitive model that combines information from the two input spaces. Input I1 contains the elements of heroine and her state of mind like grief, bitterness, sorrow and mourn. Input I2 contains the elements of boat and its anchoring and whistling. The generic space consists of a cause and effect abstract event structure that guides the mapping: the whistle of the boat may stir the emotion. There is a cross-space mapping between the elements in the input spaces, such as happiness and whistle, mourn and whistle. But the projection is partial and selective. According to the context the sentence provides, in input I1, the state of sorrow or mourn is projected, and the structure of others is not. In input $\mathrm{I} 2$, the elements of anchoring and whistling are projected. In blending, two structures from input spaces are projected to the blended space. Moreover, there is emergent structure through completion: the whistle makes people feel mournful. Consequently, the mournful whistle in blend is connected conceptually to the inputs. Bu this way, the bitterness and sadness the heroine experienced is greatly highlighted. We 
can conclude that the phrase can be counted as source input space, while target input space is introduced not form the phrase, but from the context provided by the background which can be taken as one hidden input. Based on the above analysis, this novel and specific as well as superficially illogical structure emerges. See fig. 5.

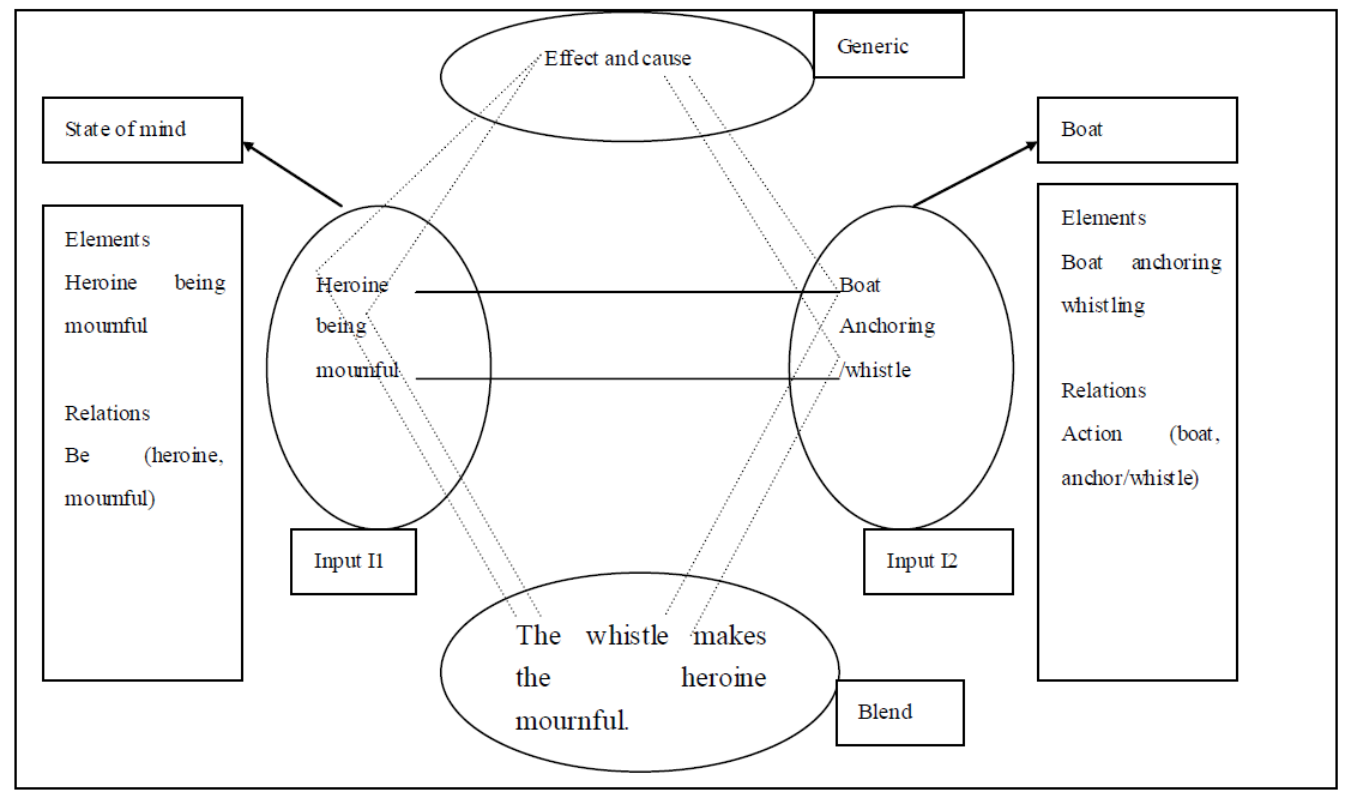

Figure 5

\section{CONCLUSION}

After careful study of meaning construction in transferred epithets by means of conceptual integration theory, we may have an overall picture of its strong force in interpreting the figure of speech - transferred epithet. I can draw the following conclusion.

Firstly, in transferred epithet, two words which do not allocate are joined together to give novel and vivid expression. It is extraordinary characteristic that we find the possibility among the impossibility. The conceptual integration theory helps us to find what is happened backstage. That is also the reason that such combination does not prevent us from understanding the speaker's intension. Secondly, the intention of writers and the contextual information around or beyond the phrase plays an important role in meaning construction of transferred epithet. Thirdly, the mapping between spaces inheres the following characteristics: 1) The cross-space mapping involves the establishment of mental spaces, and the transferred epithet is usually composed of the modifier and modified. Mostly the modified structures one input. Because conceptual integration depends on our ability to recruit relevant specific knowledge in order to establish novel mapping, as for the other space, we have to construct it according to the properties of the modifier, that is, to find the space that is closely related to the on-line meaning construction. 2) The tools of conceptual integration theory, including the cline between identity, similarity and analogy links, have proven useful in explaining the variety of complex concept combinations of transferred epithet. The generic space is often apparently structured by analogy, similarity, causative and resultant event structure suggested by linguistic and background information.

To summarize, conceptual projection is a dynamic process that cannot be adequately interpreted by a static description. And conceptual integration plays a very important role in many areas of cognition.

\section{REFERENCES}

[1] Coulson, S. (1996). Mennedez Brother Virus: Blended Spaces and Internet Humor. In Adele Goldberg (Ed.). Conceptual structure, Discourse and Language. Stanford: CSLI Publications.

[2] Coulson, S. (1997). Semantic Leaps: The Role of Frame-shifting and Conceptual Blending in Meaning Construction. New York and Cambridge: Cambridge University Press.

[3] Cuddon, J. A. (1979). A Dictionary of Literary Terms. Great Britain: W and J Mackay Limited.

[4] Fauconnier, G. (1994). Mental spaces. New York: Cambridge University Press.

[5] Fauconnier, G \& Turner, M. (1994). Conceptual Projection and Middle Spaces. Technical Report no. 9401. Department of Cognitive Science, University of California, San Diego.

[6] Fauconnier, G. (1997). Mappings in Thought and Language. Cambridge, U.K.: Cambridge University Press.

[7] Fauconnier, G \& Turner, M. (1996). Blending As a Central Process of Grammar. In Adele Goldberg (Ed.) Conceptual Structure, Discourse and Language. Standford: Center for the Study of Language and Information (CSLI) Publications. Pp 113-129.

[8] Fauconnier, G. (1998). Mental Spaces, Language Modalities and Conceptual Integration. In Michael Tomasello (Ed.). The New Psychology of Language. London: Lawrence Erlbaum Associates Publishers. Pp 251-279. 
[9] Fauconnier, G \& M. Turner. (1998). Conceptual Integration Networks. Cognitive Science 22:2. 133-187.

[10] Fauconnier, G \& M. Turner. (2003). Conceptual Integration. Shanghai: Foreign Languages.

[11] Jackendoff, R. (1983). Semantics and Cognition. Mass: MIT Press.

[12] Johnson, M. (1987). The Body in the Mind: The Bodily Basis of Meaning, Imagination and Reason. Chicago: The University of Chicago Press.

[13] Lakoff, G. (1998). Cognitive Semantics. In V. Eco (ed.). Meaning and Mental Representations. Bloomington, IN: Indiana University Press. Pp119-154.

[14] Lakoff, G. (1987). Women, Fire and Dangerous Things: What Categories Reveal About the Minds. Chicago: the University of Chicago Press.

[15] Langacker, R. W. (1990). Conceptual, Image and Symbol. Berlin: Mouton de Gruyter.

[16] Lanham, R. A. (1991). A Handlist of Rhetorical Terms. USA: University of California Press.

[17] Leech, Geoffrey. (1981). Semantics. Harmondsworth: Penguin.

[18] Levinson, S. C. (1983). Pragmatics. Cambridge University Press.

[19] Nesfield, J. C. \& Wood, F. T. (1964). Manual of English Grammar and Composition. London and Basingstoke: Macmillan Publishers Ltd.

[20] Turner, Mark \& Gilles Fauconnier. (1995). Conceptual Integration and Formal Expression. Metaphor and Symbolic Activity 10, 183-203.

[21] Wales, K. (1989). A Dictionary of Stylistics. England: Longman Group UK Ltd.

Xinmei Wang, a College English teacher for English majors in Shandong University of Finance and Economics in Jinan, has been teaching English majors for more than eight years. The courses she has taught include Advanced English, Advanced English Writing, Reading, Listening, etc. During this decade, she published several papers. Her research field is theoretical linguistics. 